\title{
miR-183 inhibits autophagy and apoptosis in gastric cancer cells by targeting ultraviolet radiation resistance-associated gene
}

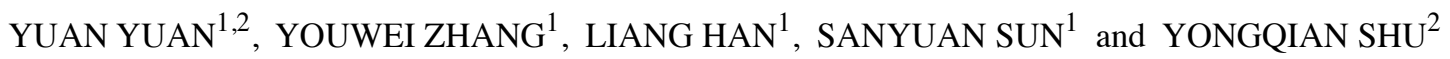 \\ ${ }^{1}$ Department of Oncology, Affiliated Xuzhou Central Hospital, Southeast University, Xuzhou, Jiangsu 221009; \\ ${ }^{2}$ Department of Oncology, First Affiliated Hospital of Nanjing Medical University, Nanjing, Jiangsu 210029, P.R. China
}

Received April 12,2018; Accepted August 21, 2018

DOI: $10.3892 /$ ijmm.2018.3871

\begin{abstract}
Ultraviolet radiation resistance-associated gene (UVRAG) regulates autophagy by promoting the formation and maturation of autophagosomes. The aim of the present study was to investigate the effects of UVRAG and UVRAG-targeting miRNA on the regulation of autophagy and apoptosis in gastric cancer (GC). TargetScan was used to predict that miR-183 targets the 3'-untranslated region (UTR) of UVRAG, while the interaction between miR-183 and the 3'-UTR of UVRAG was assessed using a dual luciferase reporter assay. Autophagy was induced in the GC cell line MKN28 by serum starvation. miR-NC mimics, miR-183 mimics, miR-NC inhibitors and miR-183 inhibitors were transfected into MKN28 cells, followed by assessment of the UVRAG expression, cell viability and cell apoptosis by western blotting, reverse transcription-quantitative polymerase chain reaction, Cell Counting Kit-8 and flow cytometry, respectively. It was demonstrated that autophagy resulted in miR-183 downregulation and overexpression of UVRAG mRNA and protein in MKN28 cells. Transfection with miR-183 mimic or inhibitor affected the expression of miR-183 and, consequently, UVRAG. miR-183 overexpression reversed the starvation-induced inhibition of cell proliferation, while inhibition of miR-183 reduced the proliferation of GC cells. miR-183 overexpression reversed starvation-induced apoptosis, while miR-183 inhibition promoted starvation-induced apoptosis in GC cells. Finally, miR-183 overexpression attenuated starvation-induced autophagy (LC-3), apoptosis (Bax/Bcl-2) and UVRAG expression, while miR-183 inhibition exerted the opposite effects. In conclusion, the results of the present study indicated that miR-183 inhibits starvation-induced autophagy and apoptosis by targeting UVRAG in human GC cells.
\end{abstract}

Correspondence to: Dr Yongqian Shu, Department of Oncology, First Affiliated Hospital of Nanjing Medical University, 300 Guangzhou Road, Gulou, Nanjing, Jiangsu 210029, P.R. China E-mail: shuyongqiannjmu@sina.com

Key words: miR-183, autophagy, apoptosis, gastric cancer, ultraviolet radiation resistance-associated gene

\section{Introduction}

Gastric cancer (GC) is the second most common cause of cancer-related mortality after lung cancer, with 738,000 deaths and 1 million new diagnoses reported annually worldwide, despite recent improvements in developed countries (1). In China, 400,000 new cases of GC are diagnosed annually, accounting for $42 \%$ of total cases worldwide (2). The tumorigenesis of GC is complex and involves the deregulation of a large number of genes $(3,4)$. Despite advances in the treatments available for $\mathrm{GC}$, the prognosis of patients with advanced-stage GC remains poor. Consequently, further research into GC progression and the related molecular mechanisms is required.

It has been reported that autophagy, which is responsible for intracellular degradation and protein recycling, plays a key role in tumor suppression and anticancer therapy (5). However, the factors that regulate the crosstalk between autophagy and apoptosis require further investigation to elucidate the mechanisms that control cell survival and death (6).

As a cDNA partially complementing ultraviolet (UV) radiation sensitivity, UV radiation resistance-associated gene (UVRAG) was first isolated from xeroderma pigmentosum cells (7), and there have also been studies that indicated the important role of UVRAG in mammalian cell autophagy $(8,9)$. UVRAG, which is a mammalian homolog of yeast Vps38, activates the Beclin-1/PI3K complex and promotes the formation of autophagosomes (10). Moreover, UVRAG has been reported to promote the maturation of autophagosomes via recruiting HOPS complexes and Rab7 to the late endosome $(11,12)$.

MicroRNAs (miRNAs) are small non-coding RNAs (22-24 nucleotides in length) that play a role in the transcriptional and post-transcriptional regulation of gene expression (13) by pairing with the $3^{\prime}$-untranslated region (UTR) of the corresponding mRNAs (14). Since the identification of lin-4 and let-7 in Caenorhabditis elegans (C.elegans), $\sim 2,000$ miRNAs have been identified, accounting for $1 \%$ of the human genome (15). miRNAs play a pivotal role in carcinogenesis; some function as oncogenes, with their overexpression leading to cancerous growth, while others act as tumor suppressors, the downregulation of which results in cancerous growth (16). Mounting evidence has revealed that miRNAs are crucial for the proliferation, apoptosis, migration, 
invasion and metabolism of tumor cells (17). Reports of miRNAs in autophagy have improved our understanding of the pathogenesis of human diseases (18), indicating that miRNAs may regulate autophagy-related genes and affect the progression of cancer.

The aim of the present study was to explore miRNAs that target UVRAG and participate in the regulation of autophagy and apoptosis in GC.

\section{Materials and methods}

Cell culture. The GC cell line MKN28 was purchased from The Institute of Biochemistry and Cell Biology at The Chinese Academy of Sciences (Shanghai, China) and grown in RPMI-1640 medium containing 10\% fetal bovine serum (FBS; Gibco; Thermo Fisher Scientific, Inc., Waltham, MA, USA) supplemented with antibiotics at $37^{\circ} \mathrm{C}$ in a humidified atmosphere containing $5 \% \mathrm{CO}_{2}$. Cells at passages 5-20 were used in the present study. To induce autophagy, MKN28 cells were rinsed with phosphate-buffered saline (PBS) twice and incubated with Hank's Balanced Salt solution (HBSS; Cellgro; Corning Incorporated, Corning, NY, USA) for $4 \mathrm{~h}$ at $37^{\circ} \mathrm{C}$ in a humidified atmosphere containing $5 \% \mathrm{CO}_{2}$, as previously described (19).

Transfection of miRNAs. miR-NC mimics, miR-183 mimics, miR-NC inhibitors or miR-183 inhibitors (Ambion; Thermo Fisher Scientific, Inc.) were transfected into MKN28 cells using Lipofectamine ${ }^{\circledR} 2000$ (Invitrogen; Thermo Fisher Scientific, Inc.) and OPTI-MEM I Reduced-Serum Medium (Invitrogen; Thermo Fisher Scientific, Inc.) in accordance with the manufacturer's instructions. The cell culture medium was changed at $24 \mathrm{~h}$ post-transfection. The following experiments were performed at $48 \mathrm{~h}$ post-transfection in triplicate.

Cell viability assay. MKN28 cells were seeded on 96-well plates at a density of $3 \times 10^{4}$ cells/well, grown in RPMI-1640 medium containing $10 \%$ fetal bovine serum (FBS), and transiently transfected with pre-miR-183 and miR-control (Ambion; Thermo Fisher Scientific, Inc.; cat. no. AM17110). At 5 days post-transfection, cell viability was detected using Cell Counting Kit-8 (CCK-8; Dojindo Molecular Technologies, Inc., Kumamoto, Japan). CCK-8 reagent $(10 \mu \mathrm{l})$ was added into each well and incubated at $37^{\circ} \mathrm{C}$ for $3 \mathrm{~h}$. Absorbance was measured at 450 and $600 \mathrm{~nm}$ using a spectrophotometer. Cell viability was evaluated using optical density (OD) as follows: Cell viability $=\mathrm{OD}_{450 \mathrm{~nm}}-\mathrm{OD}_{600 \mathrm{~nm}}$. Each experiment was performed at least in triplicate. For the detection of cell viability in the control, starvation and starvation + miR-183 mimic/inhibitor groups, cells were treated as follows: Control, cells incubated with RPMI-1640 medium containing $10 \%$ FBS; starvation, cells incubated with HBSS; starvation + miR-183 mimic/inhibitor, cells incubated with HBSS, which were transfected with miR-183 mimic/inhibitor.

Flow cytometry assay. MKN28 cells were seeded in 12-well plates and incubated for $48 \mathrm{~h}$ at $37^{\circ} \mathrm{C}$. A total of $1 \times 10^{5}$ MKN28 cells were collected by centrifugation at $1,000 \mathrm{x} \mathrm{g}$ for $5 \mathrm{~min}$ at room temperature and washed three times with PBS. Thereafter, MKN cells were fixed by pre-cooled $70 \%$ ethanol at $-20^{\circ} \mathrm{C}$ overnight. On the next day, MKN28 cells were re-suspended in RPMI-1640 medium with $10 \% \mathrm{FBS}$, mixed with Annexin V-enhanced fluorescent dye and propidium iodide (PI) and incubated for $20 \mathrm{~min}$ in the dark at room temperature. Assay results were evaluated using flow cytometry; the results were measured by Cell Quest software (BD Biosciences, Franklin Lakes, NJ, USA). For the detection of cell apoptosis in the control, starvation and starvation + miR-183 mimic/inhibitor groups, cells were treated as follows: Control, cells incubated with RPMI-1640 medium containing 10\% FBS; starvation, cells incubated with HBSS; starvation + miR-183 mimic/inhibitor, cells incubated with HBSS, which were transfected with miR-183 mimic/inhibitor.

Dual luciferase reporter assay. The potential binding site of UVRAG 3'-UTR with miRNA was predicted using TargetScan (Www.targetscan.org). miR-183 was predicted to bind with UVRAG 3'-UTR. Wild-type (wt) and mutant (mut) UVRAG 3'-UTR were synthesized and cloned into the $\mathrm{XbaI}$ site (downstream of the luciferase stop codon) of pGL3 vectors (Promega Corp., Madison, WI, USA). The resulting vectors were named pGL3-wt-UVRAG and pGL3-mut-UVRAG, respectively. 293 cells were cultured in 24-well plates and transfected with $0.4 \mathrm{mg}$ pGL3-Control, pGL3-wt-UVRAG or pGL3-mut-UVRAG with pRL-TK luciferase reporter (25 ng/well) and $20 \mathrm{~nm}$ pcDNA-miR-183 or pcDNA-miR-NC using Lipofectamine ${ }^{\circledR} 2000$. At 48 h post-transfection, a Dual-Luciferase Reporter Assay was performed to assess luciferase activity (Promega Corp.).

Western blotting. MKN28 cells were lysed using RIPA buffer containing complete protease inhibitor cocktail (Roche Molecular Diagnostics, Pleasanton, CA, USA). Protein concentrations in each sample were determined using a BCA kit (Beyotime Institute of Biotechnology, Haimen, China) in accordance with the manufacturer's instructions. Denatured proteins $(15 \mu \mathrm{g})$ were separated by $12 \%$ SDS-polyacrylamide gels and transferred to nitrocellulose membranes. Membranes were blocked in 5\% skimmed milk for $2 \mathrm{~h}$ at room temperature, followed by incubation with primary antibodies in PBS containing $0.5 \%$ Tween-20 (PBST) at $4{ }^{\circ} \mathrm{C}$ overnight. The primary antibodies were as listed: UVRAG (ABS1600; 1:1,000; Millipore, Darmstadt, Germany), BAX (50599; 1:1,000; ProteinTech Group, Inc., Chicago, IL, USA), Bcl-2 (12789; 1:1,000; ProteinTech Group, Inc.), LC3B-I/II (L8918; Sigma-Aldrich; Merck KGaA, Darmstadt, Germany) and GAPDH (5174; 1:1,000, Cell Signaling Technology, Inc.). Subsequently, the membranes were washed three times with PBST for $10 \mathrm{~min}$, followed by incubation with horseradish peroxidase (HRP)-conjugated secondary antibody (ab97080; 1:5,000; Abcam, Cambridge, MA, USA) at room temperature for $1 \mathrm{~h}$. Finally, the nitrocellulose membranes were washed three times with PBST for $10 \mathrm{~min}$ and the bands were visualized using enhanced chemiluminescence (GE Healthcare Life Science, Little Chalfont, UK) in accordance with the manufacturer's instructions. For the detection of UVRAG protein level in the control and starvation groups, the cells were treated as follows: Control, cells incubated with RPMI-1640 medium containing 10\% FBS; starvation, cells incubated with HBSS. 
For the detection of UVRAG protein level in the control, miR-NC mimic/inhibitor and miR-183 mimic/inhibitor groups, the cells were treated as follows: Control, cells incubated with RPMI-1640 medium containing 10\% FBS; miR-NC mimic/inhibitor, cells incubated with RPMI-1640 medium containing $10 \%$ FBS, which were transfected with miR-NC mimic/inhibitor; miR-183 mimic/inhibitor, cells incubated with RPMI-1640 medium containing 10\% FBS, which were transfected with miR-183 mimic/inhibitor. For the detection of BAX, Bcl-2, LC3B-I/II and UVRAG in the control, starvation and starvation + miR-183 mimic/inhibitor groups, the cells were treated as follows: Control, cells incubated with RPMI-1640 medium containing 10\% FBS; starvation, cells incubated with HBSS; starvation + miR-183 mimic/inhibitor, cells incubated with HBSS, which were transfected with miR-183 mimic/inhibitor.

$R N A$ extraction and reverse transcription-quantitative polymerasechain reaction ( $R T-q P C R)$ analysis. Total RNA was isolated from cultured MKN28 cells using TRIzol (Invitrogen; Thermo Fisher Scientific, Inc.). The integrity, quantity and purity of total RNA were determined using a Nano-Drop 8000 Spectrophotometer (Thermo Fisher Scientific, Inc.). miR-183 was reverse-transcribed and cDNA was synthesized from total RNA (10 ng) using a TaqMan ${ }^{\circledR}$ Small RNA Assay (Thermo Fisher Scientific, Inc.) in accordance with the manufacturer's instructions. Relative mRNA and miRNA levels were quantified using a mirVanaqRT-PCR miRNA Detection kit and SYBR Green I (Applied Biosystems; Thermo Fisher Scientific, Inc.). RT-qPCR was performed using an ABI Prism 7900 Sequence Detection System (Applied Biosystems; Thermo Fisher Scientific, Inc.). Subsequently, the quantification cycle $(\mathrm{Cq})$ of each sample was determined. The relative mRNA levels and miRNA levels were calculated based on the $\mathrm{Cq}$ values and $2^{-\Delta \Delta C q}$ method (20) and normalized to GAPDH or U6 level in each sample, respectively. For the detection of miR-183 and UVRAG in the control and starvation groups, cells were treated as follows: Control, cells incubated with RPMI-1640 medium containing 10\% FBS; starvation, cells incubated with HBSS. For the detection of UVRAG mRNA level and miR-183 in the control, miR-NC mimic/inhibitor, and miR-183 mimic/inhibitor groups, cells were treated as follows: Control, cells incubated with RPMI-1640 medium containing 10\% FBS; miR-NC mimic/inhibitor, cells incubated with RPMI-1640 medium containing 10\% FBS, which were transfected with miR-NC mimic/inhibitor; miR-183 mimic/inhibitor, cells incubated with RPMI-1640 medium containing $10 \%$ FBS, which were transfected with miR-183 mimic/inhibitor.

Immunofluorescence staining. MKN28 cell were seeded on glass slides in 24-well plates and incubated for $24 \mathrm{~h}$ at $37^{\circ} \mathrm{C}$, then rinsed with PBS three times. Thereafter, MKN28 cells were fixed in $4 \%$ paraformaldehyde for $30 \mathrm{~min}$ and permeabilized with $0.5 \%$ Triton $\mathrm{X}-100$ for $1 \mathrm{~h}$ at $4^{\circ} \mathrm{C}$. After blocking with $3 \%$ bovine serum albumin (Solarbio Life Sciences, Beijing, China) for $1 \mathrm{~h}$ at room temperature, the cells were incubated with rabbit anti-LC3B antibody (Sigma-Aldrich; Merck $\mathrm{KGaA}$ ) overnight at room temperature and goat anti-rabbit Alexa Fluo488 (Invitrogen; Thermo Fisher Scientific, Inc.) for $2 \mathrm{~h}$ at room temperature. The cells were then mounted onto coverslips using Prolong Gold anti-fade reagent with 4'-6-diamidino-2-phenylindole (Invitrogen; Thermo Fisher Scientific, Inc.) for $2 \mathrm{~h}$ at room temperature. Images of immunofluorescence staining in each group were captured using an AV300-ASW confocal microscope (Olympus Corp., Tokyo, Japan). For the detection of LC3 dot formation in the control, starvation and starvation + miR-183 mimic/inhibitor groups, the cells were treated as follows: Control, cells incubated with RPMI-1640 medium containing 10\% FBS; starvation, cells incubated with HBSS; starvation + miR-183 mimic/inhibitor, cells incubated with HBSS, which were transfected with miR-183 mimic/inhibitor.

Statistical analysis. Data are expressed as the mean \pm standard error of the mean. GraphPad Prism 5.0 (GraphPad Software, Inc., La Jolla, CA, USA) was used for statistical analysis. Student's t-test was used for comparisons between two groups, while multiple group comparisons were performed using one-way analysis of variance followed by Bonferroni's post hoc test. $\mathrm{P}<0.05$ was considered to indicate a statistically significant difference.

\section{Results}

UVRAG and miR-183 expression following induction of autophagy. Recent studies have revealed several autophagy-related microRNAs in cancer, including miR-183 $(21,22)$. miR-183 has clinical implications in cancer patients; for example, it was reported to be a biomarker for bladder cancer (23). To explore the potential association between miR-183 and autophagy in GC, the level of miR-183 expression was evaluated under baseline conditions and after the induction of autophagy.

As shown in Fig. 1A, RT-qPCR analysis revealed that serum starvation resulted in a distinct downregulation of miR-183 in MKN28 cells. In addition, UVRAG was identified as an mRNA target for miR-183 (Fig. 1B), and the dual luciferase reporter assay revealed that the 3'UTR of UVRAG was targeted by miR-183 (Fig. 1C). UVRAG protein and mRNA expression were increased following induction of autophagy in MKN28 cells, as demonstrated by western blotting and RT-qPCR, respectively (Fig. 1D-F). These results indicate a potential physiological function of miR-183 in GC autophagy, prompting us to further explore its precise function.

miR-183 mimics and inhibitors regulate the expression of $m i R-183$ and UVRAG. RT-qPCR was performed to assess the effects of miR-183 mimics and inhibitors on the expression of miR-183 and UVRAG in MKN28 cells. It was observed that, compared with the control and miR-NC groups, miR-183 mimics significantly upregulated the expression of miR-183, while miR-183 inhibitor significantly downregulated the expression of miR-183 (Fig. 2A and B). Compared with the control and miR-NC groups, miR-183 mimics significantly downregulated the expression of UVRAG mRNA, while miR-183 inhibitors had the opposite effect (Fig. 2C and D). The effects of miR-183 mimics and inhibitors on the expression of UVRAG protein was similar to that observed for mRNA 
B miR-183 5'-UAUGGCACUGGUAGAAUUCACU-3'
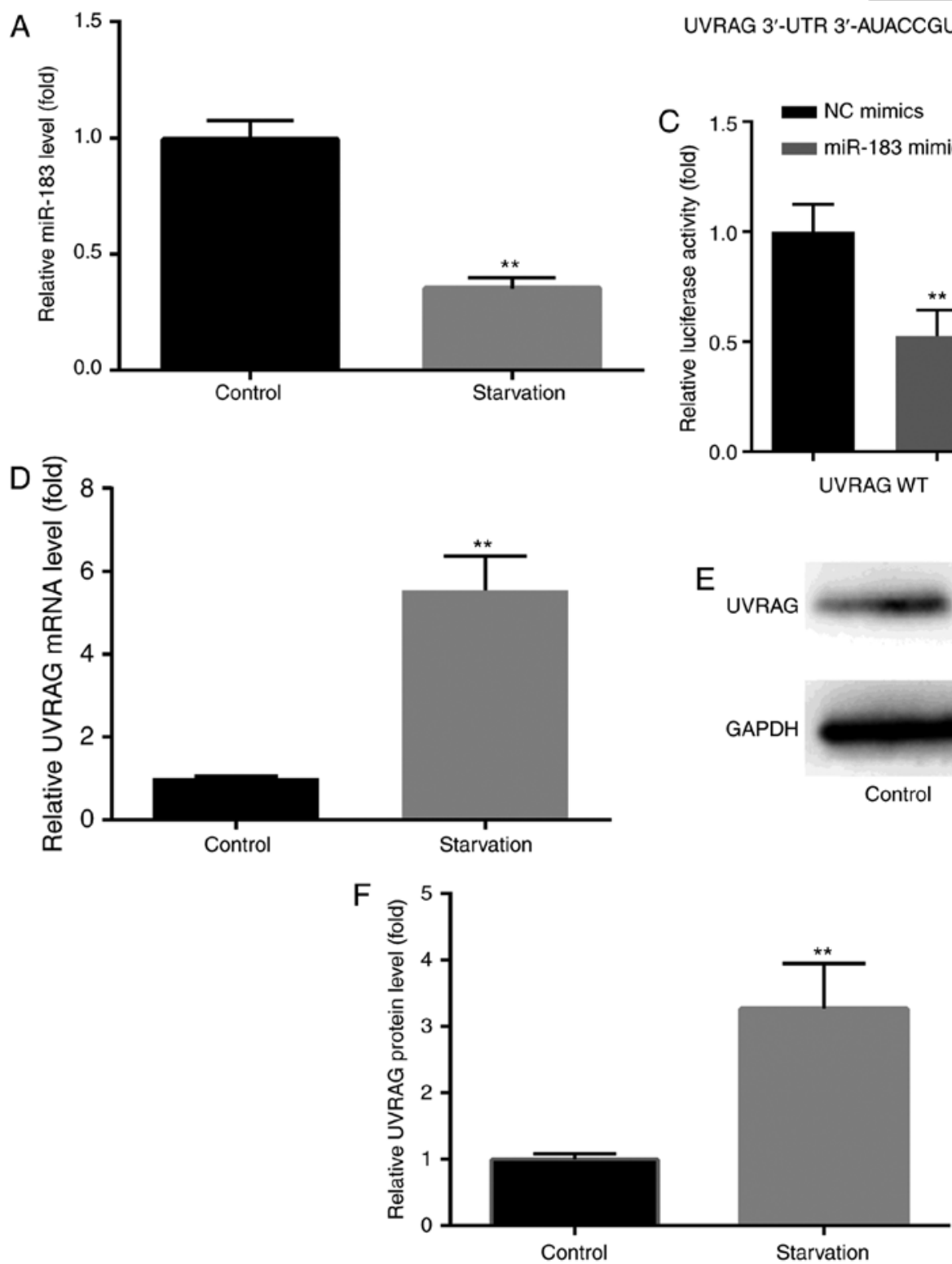

UVRAG 3'-UTR 3'-AUACCGUCUCCUCCCCUUC-5'

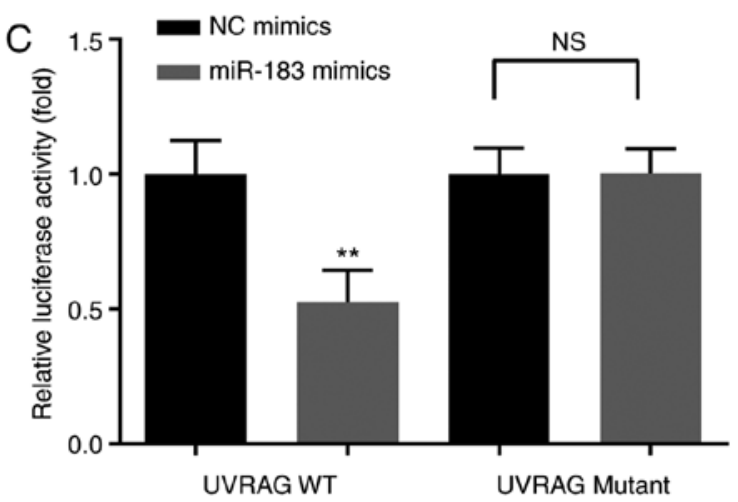

E UVRAG

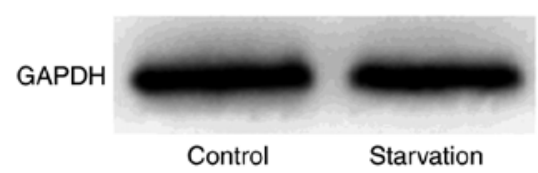

Figure 1. miR-183 downregulation and UVRAG upregulation were observed following starvation-induced autophagy. MKN28 cells were incubated in HBSS for $4 \mathrm{~h}$ (starvation conditions). (A) The relative expression of miR-183 in MKN28 cells was assessed using qPCR. (B) The miR-183 and UVRAG binding site was predicted by TargetScan. (C) The interaction between miR-183 and UVRAG was revealed using a dual luciferase assay. (D) The relative expression of UVRAG mRNA in MKN28 cells was assessed using qPCR. (E and F) The relative expression level of UVRAG protein in MKN28 cells was assessed by western blotting. $n=3 .{ }^{* *} \mathrm{P}<0.01$ starvation vs. control or miR-183 mimic vs. miR-NC mimic. Control, cells incubated with RPMI-1640 medium containing 10\% FBS; starvation, cells incubated with HBSS. UVRAG, ultraviolet radiation resistance-associated gene; HBSS, Hank's Balanced Salt solution; qPCR, quantitative polymerase chain reaction; FBS, fetal bovine serum; NC, negative control.

(Fig. 2E and F). These findings provide a new insight into the exact effects of miR-183 mimics and inhibitors on miR-183 and UVRAG expression. Transfection with miR-NC mimics or inhibitors resulted in no significant changes in miR-183 or UVRAG expression.

miR-183 regulates starvation-induced changes in cell viability and apoptosis. The effect of miR-183 on starvation-mediated cell death was assessed using CCK-8 and flow cytometry assays. Compared with the control group, starvation induction significantly downregulated cell viability; this effect was inhibited by miR-183 mimics (Fig. 3A) and enhanced by miR-183 inhibitors (Fig. 3B). Compared with the control group, starvation induction significantly upregulated cell apoptosis; this effect was reversed by miR-183 mimics (Fig. 4A and B) and augmented by miR-183 inhibitors (Fig. 4C and D). These findings may provide novel insight into the exact role of miR-183 mimics and inhibitors in starvation-induced cell viability and cell apoptosis. However, the molecules that are involved in cell apoptosis remain to be investigated.

miR-183 regulates starvation-induced changes in Bax/Bcl-2, $L C 3 B-I I / L C 3 B-I$ and UVRAG expression. We next examined the effect of miR-183 on cell death-related proteins in MKN28 cells following serum starvation. Treatment 

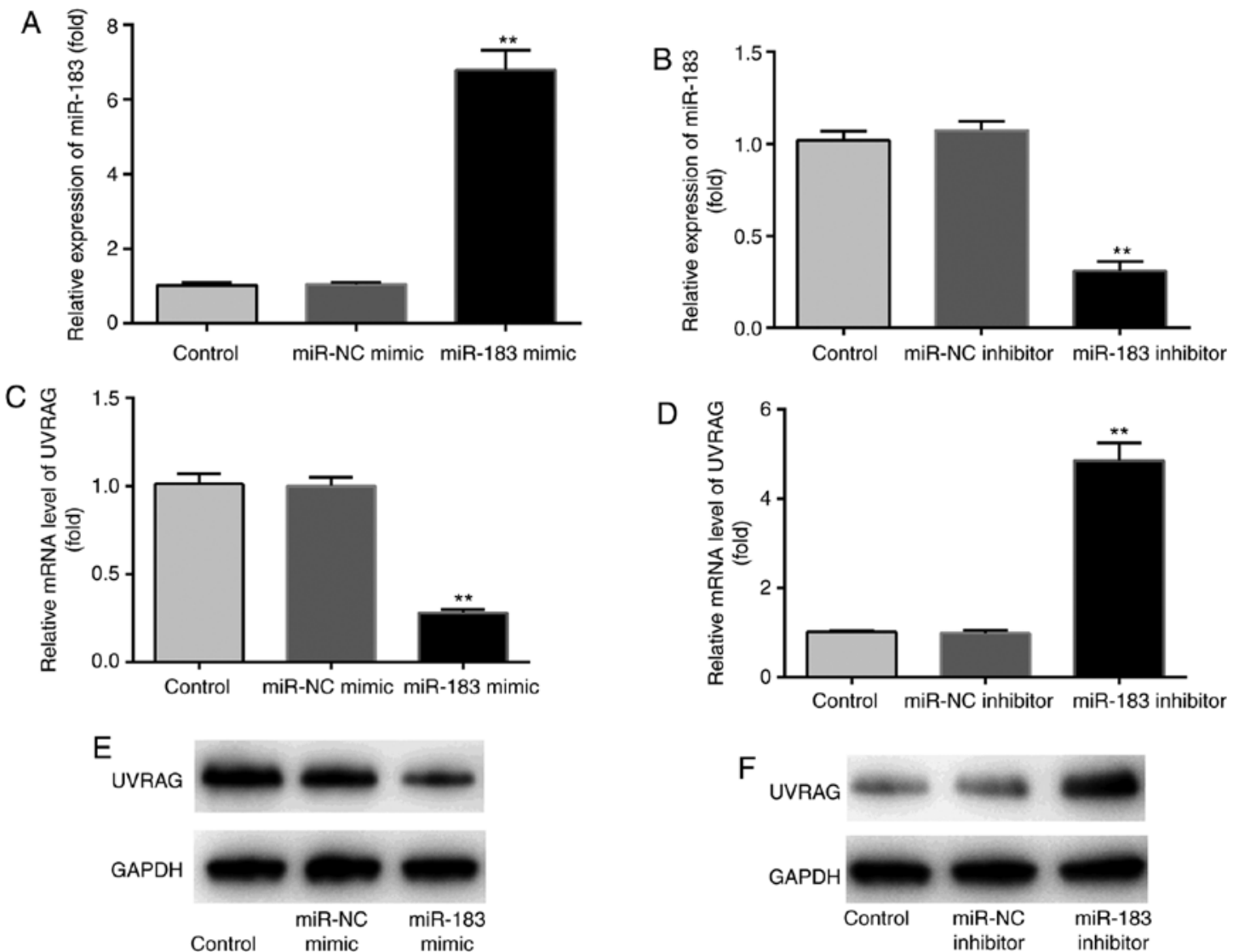

Figure 2. Effects of miR-183 mimics and inhibitors on miR-183 and UVRAG expression. Compared with the control and miR-NC groups, the expression of miR-183 was (A) significantly upregulated by miR-183 mimics and (B) downregulated by miR-183 inhibitors. Compared with the control and miR-NC groups, the expression of UVRAG mRNA was (C) significantly downregulated by miR-183 mimics and (D) significantly upregulated by miR-183 inhibitors. (E and F) miR-183 mimics and inhibitors exerted similar effects on the expression of UVRAG protein. $\mathrm{n}=3$. *" $\mathrm{P}<0.01 \mathrm{miR}-183 \mathrm{mimic}$ vs. miR-NC mimic or miR-183 inhibitor vs. miR-NC inhibitor. n.s., no significant difference. Control, cells incubated with RPMI-1640 medium containing 10\% FBS; miR-NC mimic/inhibitor, cells incubated with RPMI-1640 medium containing 10\% FBS, which were transfected with miR-NC mimic/inhibitor; miR-183 mimic/inhibitor, cells incubated with RPMI-1640 medium containing 10\% FBS, which were transfected with miR-183 mimic/inhibitor. NC, negative control; UVRAG, ultraviolet radiation resistance-associated gene; FBS, fetal bovine serum.
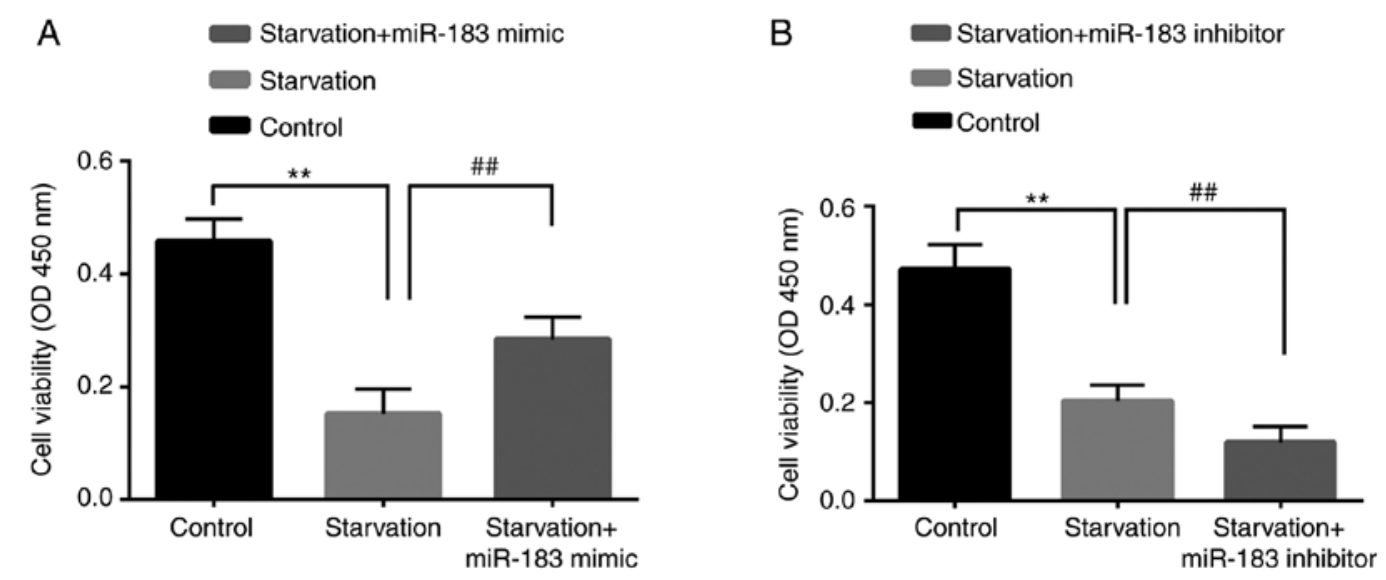

Figure 3. miR-183 regulates starvation-induced changes in cell viability. Compared with the control group, starvation induction significantly decreased cell viability, which was (A) reversed by miR-183 mimics and (B) augmented by miR-183 inhibitors. $\mathrm{n}=3$. ${ }^{* *} \mathrm{P}<0.01$ starvation vs. control; ${ }^{\# \#} \mathrm{P}<0.01$ starvation + miR-183 mimic or inhibitor vs. starvation. Control, cells incubated with RPMI-1640 medium containing 10\% FBS; starvation, cells incubated with Hank's Balanced Salt solution; starvation + miR-183 mimic/inhibitor, cells incubated with Hank's Balanced Salt solution, which were transfected with miR-183 mimic/inhibitor. FBS, fetal bovine serum.

with miR-183 mimics significantly enhanced cell survival and desensitized cells to starvation-induced apoptosis, as evidenced by decreased Bax/Bcl-2 and LC3B-II/LC3B-I ratios. Treatment with miR-183 mimics significantly reduced the starvation-induced upregulation of UVRAG (Fig. 5A-D), while treatment with miR-183 inhibitors 

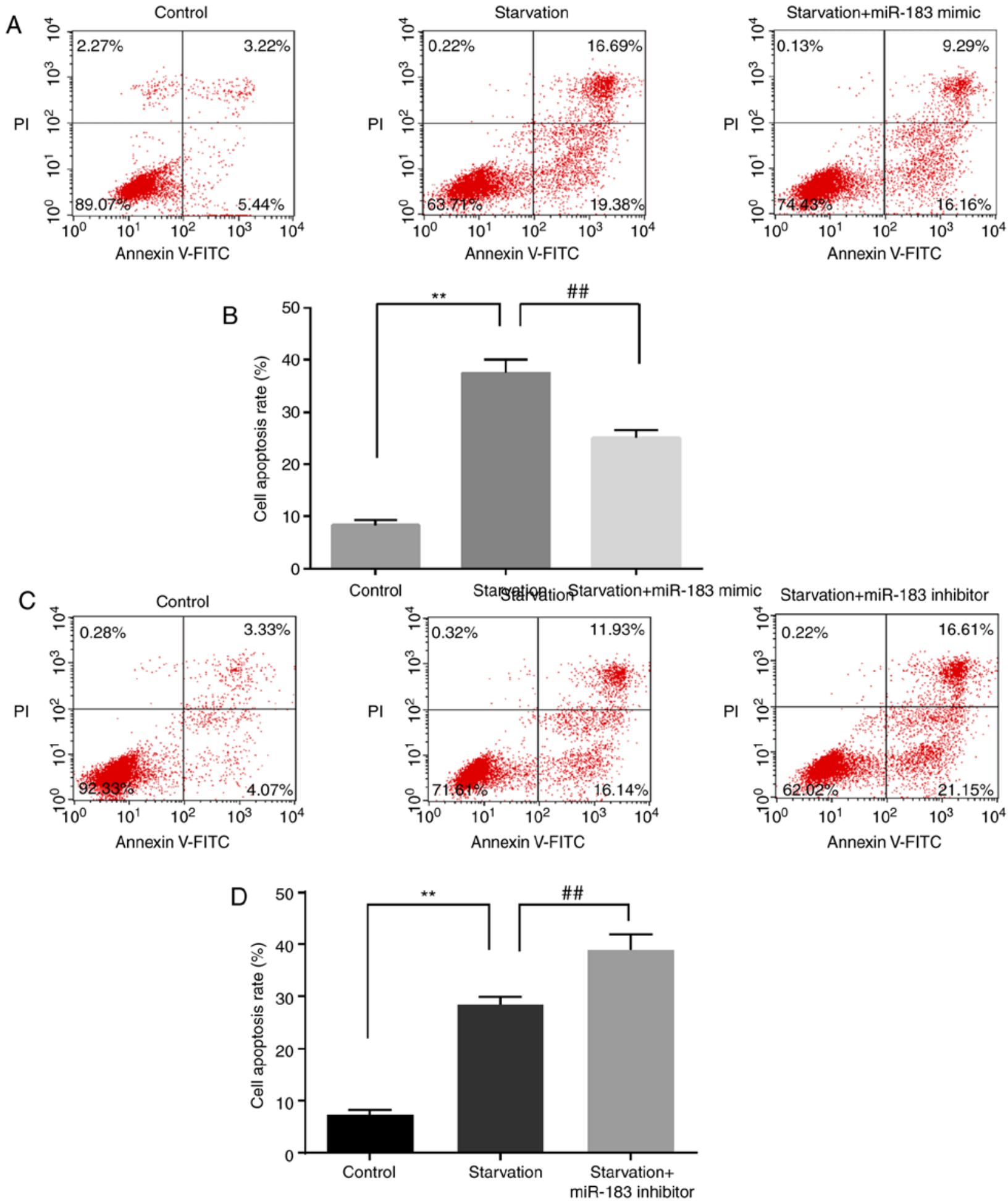

Figure 4. miR-183 regulated starvation-induced changes in cell apoptosis. Compared with the control group, starvation induction significantly upregulated cell apoptosis, which was reversed by miR-183 mimics ( $\mathrm{A}$ and $\mathrm{B}$ ) and augmented by miR-183 inhibitors (C and $\mathrm{D}) \mathrm{n}=3$. ${ }^{* *} \mathrm{P}<0.01$ starvation vs. control; ${ }^{\# \#} \mathrm{P}<0.01$ starvation + miR-183 mimic or inhibitor vs. starvation. Control, cells incubated with RPMI-1640 medium containing 10\% FBS; starvation, cells incubated with Hank's Balanced Salt solution; starvation + miR-183 mimic/inhibitor, cells incubated with Hank's Balanced Salt solution, which were transfected with miR-183 mimic/inhibitor. FBS, fetal bovine serum.

exerted the opposite effects (Fig. 5E-H). These findings may provide novel insight into the exact role of miR-183 mimics and inhibitors in starvation-induced $\mathrm{Bax} / \mathrm{Bcl}-2$, LC3B-II/LC3B-I and UVRAG expression. However, the role of miR-183 in starvation-induced autophagy remains to be investigated.
miR-183 regulates starvation-induced autophagy. The effects of miR-183 on autophagy were assessed using immunofluorescence staining. Starvation-induced LC3 dot formation (Fig. 6A and B) was reversed by miR-183 mimics (Fig. 6A) and promoted by miR-183 inhibitors in MKN28 cells (Fig. 6B). In summary, miR-183 inhibited starvation-induced autophagy. 

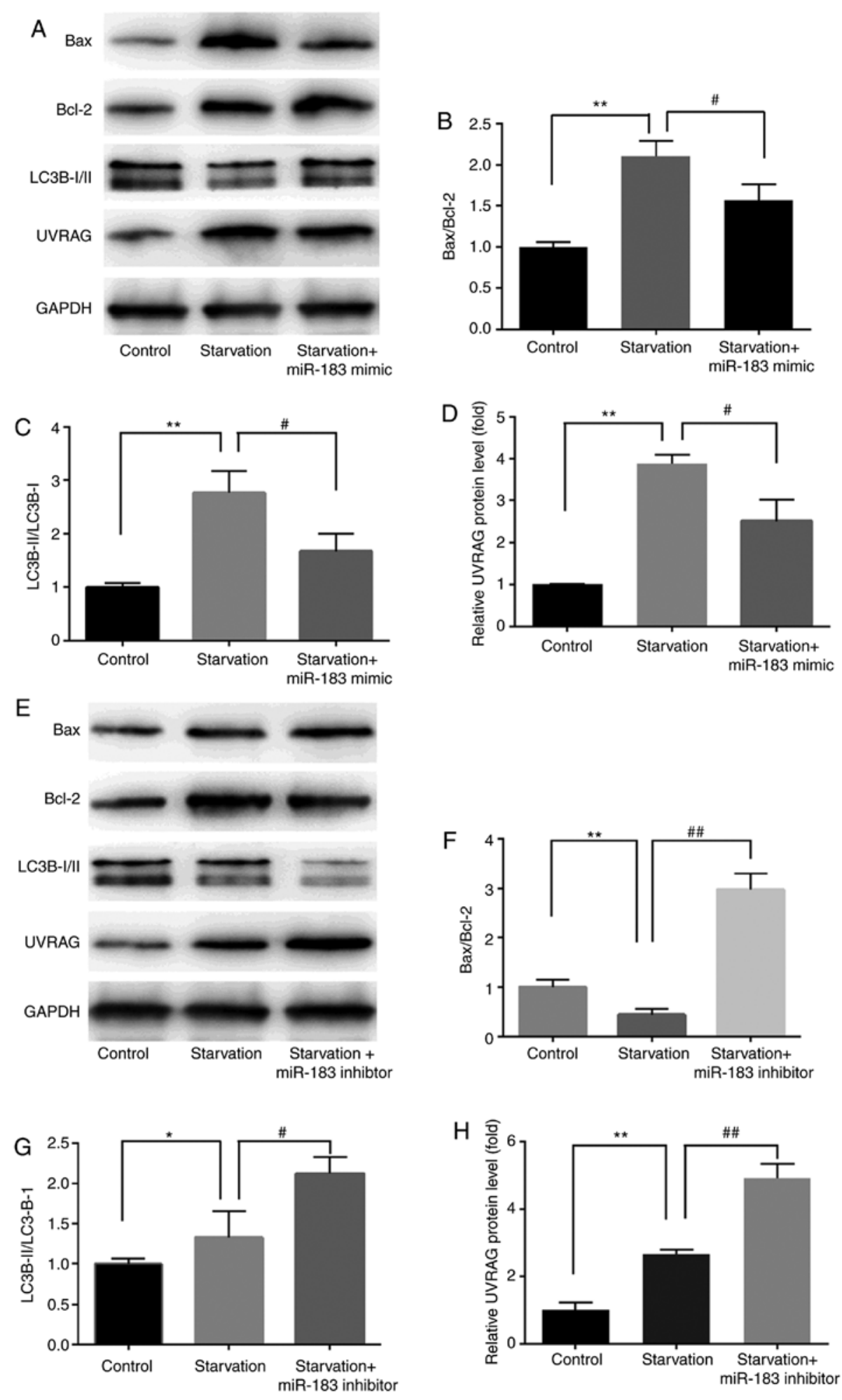

Figure 5. miR-183 regulated starvation-induced changes in Bax/Bcl-2, LC3B-II/LC3B-I and UVRAG expression. (A-D) miR-183 mimics significantly decreased the Bax/Bcl-2 and LC3B-II/LC3B-I ratios and increased UVRAG expression following serum starvation. (E-H) miR-183 inhibitors significantly increased the Bax/Bcl-2 and LC3B-II/LC3B-I ratios and decreased UVRAG expression following serum starvation. $\mathrm{n}=3 .{ }^{*} \mathrm{P}<0.05,{ }^{* *} \mathrm{P}<0.01$ starvation vs. control; ${ }^{\#} \mathrm{P}<0.05$ and ${ }^{\# \#} \mathrm{P}<0.01$ starvation + miR-183 mimic or inhibitor vs. starvation. Control, cells incubated with RPMI-1640 medium containing $10 \%$ FBS; starvation, cells incubated with Hank's Balanced Salt solution; starvation + miR-183 mimic/inhibitor, cells incubated with Hank's Balanced Salt solution, which were transfected with miR-183 mimic/inhibitor. UVRAG, ultraviolet radiation resistance-associated gene; FBS, fetal bovine serum.

\section{Discussion}

Apoptosis is established as the major mechanism of programmed cell death (PCD) and has been widely investigated (24). However, apoptosis is not the sole type of PCD; autophagy ('self-eating') plays a role in self-destructive cell progression and is intimately associated with the pathogenesis of a number of human diseases, including cell death 


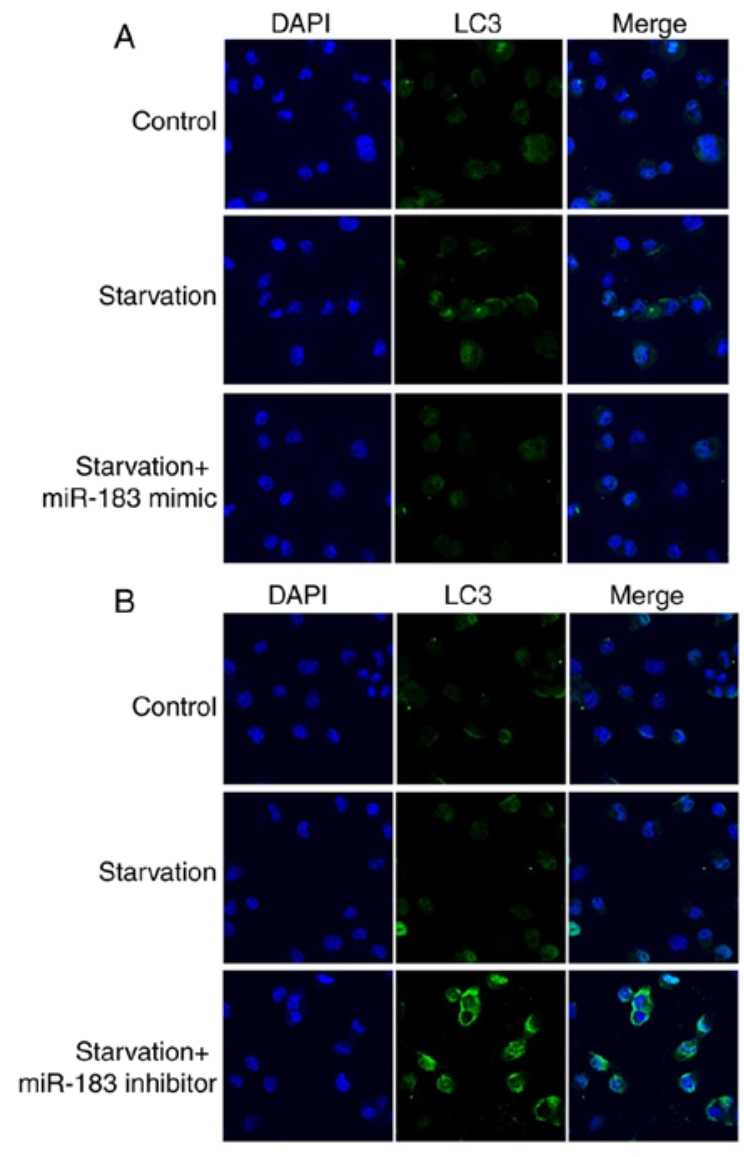

Figure 6. miR-183 regulated starvation-induced LC3 dot formation. Starvation-induced LC3 dot formation was (A) reversed by miR-183 mimics and (B) promoted by miR-183 inhibitors in MKN28 cells. Control, cells incubated with RPMI-1640 medium containing 10\% FBS; starvation, cells incubated with Hank's Balanced Salt solution; starvation + miR-183 mimic/inhibitor, cells incubated with Hank's Balanced Salt solution, which were transfected with miR-183 mimic/inhibitor. FBS, fetal bovine serum.

in GC (25). Autophagy and apoptosis commonly occur in the same cell, and autophagy precedes apoptosis (26). Autophagy triggers necrosis and stimulates cell death by apoptosis (27); however, the final cell death is achieved by autophagy but not apoptosis (28). It remains to be determined how autophagosome accumulation triggers apoptosis (29).

The role of miRNAs in autophagy may improve our understanding of the pathogenesis of multiple types of human diseases (18), as miRNAs regulate autophagy-related genes and affect the progression of cancer. For example, miR-183 has been reported to modulate autophagy and apoptosis in colorectal cancer (19). miR-183 has clinical implications in cancer patients; for example, it was reported to be a biomarker for bladder cancer (23). Furthermore, miR-183 inhibits GC cell proliferation and invasion by targeting Bmi-1 (30), while miR-183-5p is negatively correlated with the overall survival of GC patients (31). These findings suggest the potential role of miR-183 in GC and autophagy. In colorectal cancer, autophagy is an important mechanism affecting the interaction between genetic susceptibility and metabolic dysregulation, which contributes to the pathogenesis of the disease (32). Consequently, investigating the mechanism by which miR-183 and autophagy pathways are modulated may improve our understanding of GC pathogenesis.
UVRAG has recently been identified as a risk factor for colorectal cancer (33). Genotoxic and metabolic stress may result in UVRAG upregulation and apoptosis of human tumor cells (34). Surprisingly, UVRAG binds to Bax in the cytosol and prevents translocation of Bax to the mitochondria (35), suggesting that UVRAG may interact with other Bcl-2 family members to induce autophagy and trigger cell death.

LC3 is one of the biomarkers of autophagy. Once autophagy is initiated, cytosolic LC3-I converts to membrane-bound LC3-II, which is indispensable for the formation of autophagosomes (36).

In summary, the results of the present study demonstrated that miR-183 inhibits starvation-induced autophagy (LC3) and apoptosis (BAX/Bcl-2) in the human GC cell line MKN28 [identified as a derivative of the MKN74 cell line (https://web. expasy.org/cellosaurus/CVCL_1416) (37), suggesting that miR-183 acts as an autophagy-related oncogene; these findings were consistent with previous studies (19,34-36). Understanding the newly identified functions of miR-183 may allow for the development of promising therapeutic strategies for improving the clinical outcomes of cancer patients.

There were certain limitations to the present study. For example, only one cell line was used, and we plan on using at least one more cell line in our future research. Furthermore, the molecular mechanisms underlying the effects of miR-183 remain unclear and, thus, require further investigation in future studies.

\section{Acknowledgements}

Not applicable.

\section{Funding}

No funding was received.

\section{Availability of data and materials}

The data presented and analyzed are available from the corresponding author on special request.

\section{Authors' contributions}

The study was designed and data were acquired by YY, YZ, LH, SS and YS. Results interpretation was performed by YY and YZ. YY and YS wrote the manuscript. The study was supervised by YS. All authors read and approved the final manuscript

\section{Ethics approval and consent to participate}

Not applicable.

\section{Patient consent for publication}

Not applicable.

\section{Competing interests}

The authors declare that they have no competing interests. 


\section{References}

1. Jemal A, Bray F, Center MM, Ferlay J, Ward E and Forman D: Global cancer statistics. CA Cancer J Clin 61: 69-90, 2011.

2. Yang L: Incidence and mortality of gastric cancer in China. World J Gastroenterol 12: 17-20, 2006.

3. Houghton J, Stoicov C, Nomura S, Rogers AB, Carlson J, Li H, Cai X, Fox JG, Goldenring JR and Wang TC: Gastric cancer originating from bone marrow-derived cells. Science 306 : 1568-1571, 2004.

4. Zheng L, Wang L, Ajani J and Xie K: Molecular basis of gastric cancer development and progression. Gastric Cancer 7: 61-77, 2004.

5. Rubinsztein DC, Codogno P and Levine B: Autophagy modulation as a potential therapeutic target for diverse diseases. Nat Rev Drug Discov 11: 709-730, 2012.

6. Kroemer G and Levine B: Autophagic cell death: The story of a misnomer. Nat Rev Mol Cell Biol 9: 1004-1010, 2008.

7. Perelman B, Dafni N, Naiman T, Eli D, Yaakov M, Feng TL, Sinha S, Weber G, Khodaei S, Sancar A, et al: Molecular cloning of a novel human gene encoding a $63-\mathrm{kDa}$ protein and its sublocalization within the 11q13 locus. Genomics 41: 397-405, 1997.

8. Yang Y, Quach C and Liang C: Autophagy modulator plays a part in UV protection. Autophagy 12: 1677-1678, 2016.

9. Takahashi Y, Coppola D, Matsushita N, Cualing HD, Sun M, Sato Y, Liang C, Jung JU, Cheng JQ, Mulé JJ, et al: Bif-1 interacts with Beclin 1 through UVRAG and regulates autophagy and tumorigenesis. Nat Cell Biol 9: 1142-1151, 2007.

10. Liang C, Feng P, Ku B, Oh BH and Jung JU: UVRAG: A new player in autophagy and tumor cell growth. Autophagy 3: 69-71, 2007.

11. McKnight NC, Zhong Y, Wold MS, Gong S, Phillips GR, Dou Z, Zhao Y, Heintz N, Zong WX and Yue Z: Beclin 1 is required for neuron viability and regulates endosome pathways via the UVRAG-VPS34 complex. PLoS Genet 10: e1004626, 2014

12. Liang C, Lee JS, Inn KS, Gack MU, Li Q, Roberts EA, Vergne I, Deretic V, Feng P, Akazawa C and Jung JU: Beclin1-binding UVRAG targets the class C Vps complex to coordinate autophagosome maturation and endocytic trafficking. Nat Cell Biol 10: 776-787, 2008

13. Bartel DP: MicroRNAs: Genomics, biogenesis, mechanism, and function. Cell 116: 281-297, 2004.

14. Lee Y, Ahn C, Han J, Choi H, Kim J, Yim J, Lee J, Provost P, Rådmark O, Kim S and Kim VN: The nuclear RNase III Drosha initiates microRNA processing. Nature 425: 415-419, 2003.

15. Lee RC, Feinbaum RL and Ambros V: The C. elegans heterochronic gene lin-4 encodes small RNAs with antisense complementarity to lin-14. Cell 75: 843-854, 1993.

16. Lawrie $\mathrm{CH}$ : MicroRNAs and haematology: Small molecules, big function. Br J Haematol 137: 503-512, 2007.

17. Lu J, Getz G, Miska EA, Alvarez-Saavedra E, Lamb J, Peck D, Sweet-Cordero A, Ebert BL, Mak RH, Ferrando AA, et al MicroRNA expression profiles classify human cancers. Nature 435: 834-838, 2005 .

18. Frankel LB and Lund AH: MicroRNA regulation of autophagy. Carcinogenesis 33: 2018-2025, 2012.

19. Huangfu L, Liang H, Wang G, Su X, Li L, Du Z, Hu M, Dong Y, Bai X, Liu T, et al: miR-183 regulates autophagy and apoptosis in colorectal cancer through targeting of UVRAG. Oncotarget 7: $4735-4745,2016$
20. Livak KJ and Schmittgen TD: Analysis of relative gene expression data using real-time quantitative PCR and the 2(-Delta Delta $\mathrm{C}(\mathrm{T}))$ method. Methods 25: 402-408, 2001.

21. Pan B, Yi J and Song H: MicroRNA-mediated autophagic signaling networks and cancer chemoresistance. Cancer Biother Radiopharm 28: 573-578, 2013.

22. Abraham D, Jackson N, Gundara JS, Zhao J, Gill AJ, Delbridge L, Robinson BG and Sidhu SB: MicroRNA profiling of sporadic and hereditary medullary thyroid cancer identifies predictors of nodal metastasis, prognosis, and potential therapeutic targets. Clin Cancer Res 17: 4772-4781, 2011.

23. Nagata M, Muto S and Horie S: Molecular biomarkers in bladder cancer: Novel potential indicators of prognosis and treatment outcomes. Dis Markers 2016: 8205836, 2016.

24. Liu G, Pei F, Yang F, Li L, Amin AD, Liu S, Buchan JR and Cho WC: Role of autophagy and apoptosis in non-small-cell lung cancer. Int J Mol Sci 18: E367, 2017.

25. Qian HR and Yang Y: Functional role of autophagy in gastric cancer. Oncotarget 7: 17641-17651, 2016.

26. Maiuri MC, Zalckvar E, Kimchi A and Kroemer G: Self-eating and self-killing: Crosstalk between autophagy and apoptosis. Nat Rev Mol Cell Biol 8: 741-752, 2007.

27. Galluzzi L, Vitale I, Abrams JM, Alnemri ES, Baehrecke EH, Blagosklonny MV, Dawson TM, Dawson VL, El-Deiry WS, Fulda S, et al: Molecular definitions of cell death subroutines: Recommendations of the nomenclature committee on cell death 2012. Cell Death Differ 19: 107-120, 2012.

28. Shen HM and Codogno P: Autophagic cell death: Loch Ness monster or endangered species? Autophagy 7: 457-465, 2011.

29. Ma X, Liu H, Foyil SR, Godar RJ, Weinheimer CJ, Hill JA and Diwan A: Impaired autophagosome clearance contributes to cardiomyocyte death in ischemia/reperfusion injury. Circulation 125: 3170-3181, 2012.

30. Xu L, Li Y, Yan D, He J and Liu D: MicroRNA-183 inhibits gastric cancer proliferation and invasion via directly targeting Bmi-1. Oncol Lett 8: 2345-2351, 2014.

31. Li CY, Liang GY, Yao WZ, Sui J, Shen X, Zhang YQ, Peng H, Hong WW, Ye YC, Zhang ZY, et al: Identification and functional characterization of microRNAs reveal a potential role in gastric cancer progression. Clin Transl Oncol 19: 162-172, 2017.

32. He C and Klionsky DJ: Regulation mechanisms and signaling pathways of autophagy. Annu Rev Genet 43: 67-93, 2009.

33. Goi T, Kawasaki M, Yamazaki T, Koneri K, Katayama K, Hirose K and Yamaguchi A: Ascending colon cancer with hepatic metastasis and cholecystolithiasis in a patient with situs inversus totalis without any expression of UVRAG mRNA: Report of a case. Surg Today 33: 702-706, 2003.

34. Zhao Z, Ni D, Ghozalli I, Pirooz SD, Ma B and Liang C: UVRAG: At the crossroad of autophagy and genomic stability. Autophagy 8: 1392-1393, 2012.

35. Yin X, Cao L, Kang R, Yang M, Wang Z, Peng Y, Tan Y, Liu L, Xie M, Zhao Y, et al: UV irradiation resistance-associated gene suppresses apoptosis by interfering with BAX activation. EMBO Rep 12: 727-734, 2011.

36. Lee J, Giordano S and Zhang J: Autophagy, mitochondria and oxidative stress: Cross-talk and redox signalling. Biochem J 441: 523-540, 2012.

37. Motoyama T, Hojo $\mathrm{H}$ and Watanabe $\mathrm{H}$ : Comparison of seven cell lines derived from human gastric carcinomas. Acta Pathol Jpn 36: 65-83, 1986. 